\title{
La identidad profesional en estudiantes y docentes desde el contexto universitario: una revisión
}

\author{
The professional identity in students and university professors \\ in a university context: a review \\ * Paulina Elizabeth González Orozco, ** Rigoberto Marín Uribe, \\ ** Ma. Concepción Soto Valenzuela
}

González P., Marín, R. \& Soto, MA. (2019). La identidad profesional en estudiantes y docentes desde el contexto universitario: una revisión. Revista Ciencias de la Actividad Física UCM, N²0(1), enero-junio, 1-14.

DOI: http://doi.org/10.29035/rcaf.20.1.4

\section{RESUMEN}

En el trayecto formativo de todo profesional se desarrollan procesos de construcción identitaria, en ellos concurren diversos componentes, siendo el entorno un elemento necesario para comprender la adquisición y evolución de la identidad. Este artículo busca analizar los resultados de una revisión sistemática realizada sobre la construcción de la identidad profesional. En este estudio se examinaron 20 investigaciones que recuperan los conceptos y metodologías utilizadas, así como los principales hallazgos reportados en estudios que pretenden comprender el proceso de construcción de la identidad profesional en estudiantes y docentes dentro del contexto universitario, la revisión abarca documentos comprendidos entre los años 2006 a 2015; con base en la revisión se ofrece una concepción de identidad profesional y se encuentra que la metodología con enfoque cualitativo es la que mejor responde a las problemáticas planteadas en estos estudios.

\section{PALABRAS CLAVE}

Identidad profesional, formación universitaria, representación, metodología.

\begin{abstract}
The curriculum of every professional processes for constructive identity is developed through various components, being that the environment is a necessary element to understand the acquisition and evolution of identity. The aim of the study is to analyze the results of a systematic review carried out on the construction of the professional identity. In this study we examined 20 investigations that retrieve the concepts and methodologies used, as well as the main findings reported in studies that pretend to understand the construction process of the professional identity in students and university professors within the university context. This review covers documents published between the years 2006 to 2015; based on the revision that offers a conception of professional identity and it is found that methodology that uses a qualitative approach best responds to the issues raised in these studies.
\end{abstract}

Key words

Professional identity, university formation, representation, methodology.

* Maestría Universidad Autónoma de Chihuahua, México.

** Doctorado Universidad Autónoma de Chihuahua, México. 


\section{INTRODUCCIÓN}

La identidad es un proceso dinámico y activo que sirve para asumir una idea de sí mismo y de las representaciones que se construyen al estar inmerso en una comunidad, puesto que se construye dentro de la interdependencia respecto de los grupos sociales de los que forma parte, teniendo al propio sujeto como un protagonista importante, configurando la interrelación entre sujeto, sociedad y cultura (Hirsch, 2013); de tal manera que durante el trayecto formativo, la vida escolar permite al sujeto remitirse a referentes individuales y colectivos que habrán de aportar elementos importantes para la configuración de su propia identidad.

Puesto que la identidad implica un modo de sentir, comprender y actuar en el mundo en donde se integran factores sociales y personales; hablamos de una representación autorreferencial formada por atributos cognitivos y emocionales que se perciben como propios y que dan cierta estabilidad y continuidad a la vida profesional (Monereo \& Domínguez, 2014), al respecto, Navarrete (2008), señala que la identidad profesional, "es la forma en que un sujeto se apropia de un proyecto profesional-institucional, correspondiente a un campo disciplinar, y de lo que el proyecto y ese campo implican en tanto espacio y medio de constitución-formación" (p.146); en este sentido, se destaca la importancia que el contexto educativo representa como un espacio de transformación del individuo, brindando la oportunidad tanto a estudiantes como profesores, de adquirir las competencias fundamentales para apropiarse de su profesión y con ello, avanzar en el proceso de construcción de su identidad (Barrenetxea, Cardona, Barandiaran, Mijangos \& Olaskoaga, 2013; Zanatta, Yurén \& Faz, 2010).

La identidad representa un proceso dinámico que evoluciona con el tiempo y que se reconstruye constantemente derivando en un concepto profundo que no es inmutable, al mismo tiempo, la comprensión de cómo los individuos adquieren y desarrollan procesos de construcción identitaria resulta igualmente compleja; de todo esto se desprende la impor- tancia de recuperar aquellos estudios que han contribuido al entendimiento de la identidad profesional como resultado de un proceso de formación continua relacionada con el contexto educativo, tanto lo que representa para los estudiantes como para los docentes. Por esta razón, surge la necesidad de responder a la interrogante ¿Cómo se da el proceso de construcción y conceptualización de la identidad profesional en estudios realizados dentro del contexto educativo?, siendo más específicos en el nivel universitario.

Bajo esta perspectiva, el presente trabajo tiene como objetivo comprender cómo se significa y estudia el concepto de identidad profesional en el contexto universitario, realizando para ello una revisión sistemática de artículos científicos que han sido publicados en la última década, entre los años 2006 y 2015, orientando el análisis hacia 4 elementos: 1) Objetivos perseguidos en las investigaciones revisadas, 2) Metodología utilizada, definiendo el tipo de estudio y las herramientas para recolección de datos, 3) Principales resultados y 4) Identificación de las características en torno al concepto de identidad que manejan los autores.

Adicionalmente, sobre el análisis presentado se ofrece una conceptualización de la definición de identidad que busca rescatar los principales elementos que confluyen en la construcción identitaria profesional.

\section{METODOLOGÍA}

Para responder a la necesidad planteada, se realizó una búsqueda sistemática de artículos científicos en internet relacionados con el tema de interés: la identidad profesional en el contexto universitario; eligiendo aquellos contenidos en las bases de datos ERIC, EBSCO, ELSEVIER y SCIELO, se establecieron como palabras clave los conceptos: identity, professional, university y formation. Los criterios de selección fueron: 1) artículos publicados en revistas científicas durante el periodo 20062015, 2) artículos sin restricción de idiomas y 3) artículos en los que al menos una de las palabras clave apareciera en el título. 
Una vez seleccionados los artículos que cumplían con los criterios establecidos, se retomaron aquellos que estuvieran en la modalidad de open Access, que fueran escritos en idioma inglés, español o portugués y que su población de estudio fueran estudiantes $\mathrm{y} / \mathrm{o}$ docentes en el nivel universitario, finalmente, se seleccionaron un total de veinte artículos, los cuales, de acuerdo a su población, fueron clasificados por sujetos de estudio en estudiantes (12 artículos) y docentes (8 artículos).

Para el análisis de los artículos seleccionados se consideraron las vertientes antes mencionadas, describiendo así, los objetivos planteados en cada estudio, la metodología utilizada, los principales resultados, la caracterización precisada del concepto de identidad, así como, la contextualización de donde surgen estos estudios.

\section{RESULTADOS Y DISCUSIÓN}

Para un mejor entendimiento del análisis de los artículos seleccionados, a continuación se exponen los principales aspectos reconocidos de investigaciones realizadas en estudiantes durante su formación universitaria; presentando a los autores y el contexto donde se desarrollaron sus estudios en un esquema dividido en dos partes para facilitar su entendimiento. En primer término y en concordancia con los primeros elementos perseguidos en este estudio, se presentan en la Tabla 1 los objetivos y la metodología empleada por los autores que se citan en la misma, posteriormente se señalan los aspectos más relevantes de los mismos.

\section{Tabla 1}

Investigaciones de la identidad profesional en estudiantes: objetivos y metodología.

\begin{tabular}{lll}
\hline \multicolumn{1}{c}{ Autor, año, contexto } & \multicolumn{1}{c}{ Objetivos } & $\begin{array}{c}\text { Tipo de estudio/Herramientas para } \\
\text { recolección de datos }\end{array}$ \\
\hline $\begin{array}{l}\text { Sayago, Chacón \& } \\
\text { Rojas. 2008. }\end{array}$ & $\begin{array}{l}\text { Analizar los referentes de identidad } \\
\text { construidos durante la formación uni- } \\
\text { Venezuela. }\end{array}$ & $\begin{array}{l}\text { Investigación cualitativa con enfoque } \\
\text { biográfico narrativo/Biogramas, rela- } \\
\text { tos autobiográficos y entrevistas. }\end{array}$ \\
\hline $\begin{array}{l}\text { Bedacarratx. 2012. } \\
\text { Argentina. }\end{array}$ & $\begin{array}{l}\text { "Indagar los significados y procesos } \\
\text { que se ponen en juego en la constitu- } \\
\text { ción de la identidad profesional". }\end{array}$ & $\begin{array}{l}\text { Metodología cualitativa con enfoque } \\
\text { interpretativo/.Entrevistas en profun- } \\
\text { didad y semiestructuradas, observa- } \\
\text { ciones de clases, sesiones de grupos de } \\
\text { reflexión y diarios de campo. }\end{array}$ \\
\end{tabular}

Navarrete. 2008.

México.
Determinar los rasgos que han definido la identidad de los pedagogos de la UNAM y UV egresados en 1950, 1970 y 1990.

Sugimura \& Shimizu. Explorar el desarrollo de la identidad 2011. Japón. en estudiantes japoneses en una esfera de aprendizaje en transición a la universidad.

Balduzzi \& Egle. 2010. "Identificar indicios de la construcción México. de la identidad profesional en estudiantes universitarios avanzados".
Investigación cualitativa con análisis histórico-documental/Revisión planes de estudio y entrevistas.

Investigación exploratoria con aplicación del test McNemar en dos tiempos y el análisis de narrativa/Programa de intervención. Narrativas

Metodología cualitativa con un diseño de tipo exploratorio-descriptivo/Entrevistas semiestructuradas y grupos focales.

Limberg et al. 2013. Identificar las experiencias específicas USA. que influyen en el desarrollo de la identidad profesional.

Timostsuk \& Ugaste. 2010. Estonia.
Investigación cualitativa consensual: fenomenológica, análisis completo y teoría fundamentada/Grupos focales, entrevistas semiestructuradas.

Investigación cualitativa. Entrevistas semiestructuradas y entrevistas a grupos focales. Programa Nvivo 7 para el análisis de datos. 


\begin{tabular}{|c|c|c|}
\hline Autor, año, contexto & Objetivos & $\begin{array}{l}\text { Tipo de estudio/Herramientas para } \\
\text { recolección de datos }\end{array}$ \\
\hline $\begin{array}{l}\text { Kim, Copeland, } \\
\text { Hopson, Simmons \& } \\
\text { Leibowitz. 2013. USA. }\end{array}$ & $\begin{array}{l}\text { Examinar si la identidad es un predic- } \\
\text { tor del éxito en la escuela de posgrado } \\
\text { y si difiere entre estudiantes matricula- } \\
\text { dos y no matriculados. }\end{array}$ & $\begin{array}{l}\text { Enfoque mixto: investigación cuali- } \\
\text { tativa y cuantitativa/SACNAS (Escala } \\
\text { científica de la identidad). entrevistas } \\
\text { semiestructuradas }\end{array}$ \\
\hline Gur. 2014. Turquía. & $\begin{array}{l}\text { Investigar como los candidatos a maes- } \\
\text { tros configuran el desarrollo de su } \\
\text { identidad profesional, entendiendo el } \\
\text { cómo perciben y eligen su profesión. }\end{array}$ & $\begin{array}{l}\text { Estudio cualitativo. Análisis crítico del } \\
\text { discurso/Grupos de discusión video } \\
\text { grabados. Entrevistas. }\end{array}$ \\
\hline $\begin{array}{l}\text { Kovalcikiene \& } \\
\text { Buksnyte-Marmiene. } \\
\text { 2015. Lituania. }\end{array}$ & $\begin{array}{l}\text { Analizar las peculiaridades de la iden- } \\
\text { tidad profesional de los estudiantes de } \\
\text { doctorado. }\end{array}$ & $\begin{array}{l}\text { Enfoque descriptivo/Cuestionario de } \\
\text { identidad profesional en estudiantes de } \\
\text { doctorado. }\end{array}$ \\
\hline $\begin{array}{l}\text { Pardal, Neto-Mendes, } \\
\text { Martins \& Goncalves. } \\
\text { 2015. Portugal. }\end{array}$ & $\begin{array}{l}\text { Conocer, analizar y discutir las repre- } \\
\text { sentaciones sociales que los estudiantes } \\
\text { de nivel superior tienen de la identidad } \\
\text { del profesor }\end{array}$ & $\begin{array}{l}\text { Estudio cualitativo. Análisis de conte- } \\
\text { nido/Asociaciones libres. }\end{array}$ \\
\hline $\begin{array}{l}\text { Zare-ee \& Ghasedi. } \\
\text { 2014. Irán. }\end{array}$ & $\begin{array}{l}\text { Analizar los aspectos de identidad pro- } \\
\text { fesional en estudiantes de inglés como } \\
\text { lengua extranjera que planean ser futu- } \\
\text { ros profesores en Irán. }\end{array}$ & $\begin{array}{l}\text { Estudio descriptivo transversal/ Cues- } \\
\text { tionario TPI (Teachers' Professional } \\
\text { Identity). Grupos focales. }\end{array}$ \\
\hline
\end{tabular}

Fuente: elaboración propia.

Con base en lo expuesto es que, se reconocen los múltiples aspectos que entran en juego dentro de la configuración identitaria, en donde, la mayoría de los estudios presentados buscan darle significado a los rasgos de la propia identidad y a las experiencias que se van dando durante la formación académica, de este modo, se pueden comprender las representaciones sociales que constituyen una forma de pensamiento que surge dentro de un grupo determinado y que expresa las peculiaridades del mismo; de tal manera que la percepción que los estudiantes tienen respecto a su profesión se ve reflejada, dando un entendimiento mayor hacía la elección de la misma y hacía el desarrollo de la identidad profesional.

Es por lo anterior, la importancia de rescatar las experiencias de vida de los sujetos a través del entendimiento de la percepción que de sus profesiones tienen, si existe claridad en el quehacer profesional, habrá un mayor acercamiento a la representación social significando el sentido de su profesión; de modo que la investigación cualitativa es la que mayor herramientas proporciona para comprender un fenómeno tan complejo como el de la identidad profesional, si bien es cierto se observan diferentes alternativas para la recolección de datos dentro de las investigaciones orientadas en estudiantes, tanto que se habla de revisión de documentos, hasta del uso de algún test; son las entrevistas y los grupos focales quienes tienen mayor presencia en las opciones presentadas para la recolección de datos teniendo en el enfoque biográfico narrativo, interpretativo y descriptivo las elecciones naturales para su análisis.

Cabría preguntarse, de los enfoques anteriormente mencionados, cual es el que ofrece un mejor análisis para determinar los factores que permiten comprender el proceso de construcción de la identidad profesional, aunque si se observa la tendencia de las herramientas utilizadas para la recolección de datos, resulta innegable la necesidad de utilizar diferentes exponentes de información para obtener una mejor comprensión.

A continuación se presentan los principales resultados obtenidos en los mismos estudios y las características en torno al concepto de identidad que manejan los autores (Tabla 2). 
Tabla 2

Investigaciones de la identidad profesional en estudiantes: resultados y concepto de identidad.

\begin{tabular}{ll}
\hline \multicolumn{1}{c}{ Autor, año, contexto } & \multicolumn{1}{c}{ Principales resultados } \\
\hline Sayago, Chacón & Se asume la identidad como un proceso \\
\& Rojas. 2008. & individual y colectivo que se construye y \\
Venezuela. & $\begin{array}{l}\text { reconstruye con base a interacciones socio- } \\
\text { culturales. }\end{array}$
\end{tabular}

Concepto identidad

La identidad es un concepto que permite explicar los aspectos internos del sujeto y su relación con el contexto, formándose de una dimensión antropológica, psicológica y sociológica.

Bedacarratx. 2012. En el proceso de configuración identitaria Argentina.

se posiciona al docente en diferentes lugares imaginarios, se analizan un conjunto de sentimientos ambivalentes por el impacto que supone el contacto con el objeto real de trabajo.

Navarrete. 2008.

México.

La carrera del pedagogo se ha constituido de manera hibrida por lo que, se dificulta hablar de su identidad profesional en términos homogéneos, unitarios y propios de la misma, no es una identidad autocontenida en y desde sí misma.

Sugimura \& Shimizu. 2011. Japón.

El papel de los compañeros puede ser un componente eficaz en un programa de intervención para promover el desarrollo de la identidad del adolescente a través del aprendizaje cooperativo.

Balduzzi \& Egle. 2010. En la construcción de la identidad profeMéxico. sional se resalta la confrontación entre las expectativas determinadas en las creencias y significados que el estudiante otorga, en contraste con las primeras impresiones adquiridas en el ejercicio de su profesión.

Limberg et al. 2013.

USA.

La influencia de la facultad y la creación de redes como las tutorías y conferencias son relevantes para el desarrollo de la identidad profesional.

Timostsuk \& Ugaste. 2010. Estonia.
La identidad es la representación que un sujeto tiene de sí mismo en su percepción individual o colectiva.

La identidad profesional es la forma en la que el sujeto se apropia de un proyecto profesional-institucional dentro de un campo disciplinar, dependiendo de la manera en la que se relaciona y se forma dentro de un contexto especifico.

La identidad surge a partir de las interacciones que el propio sujeto desarrolle, de los valores y creencias propios y de los contextos socioculturales en los que se vea expuesto.

La identidad es asumida como un proceso constante y continuo que se determina por la relación entre el entorno laboral y el individuo que se asume a sí mismo como parte de un gremio durante su actuar profesional.

La identidad profesional se define como la integración de los atributos personales y la formación profesional en el contexto de una comunicad profesional.

La identidad del profesional es el autoconocimiento de la persona en situaciones e interacciones relacionadas con la práctica profesional, los sentimientos de pertenencia y las experiencias de aprendizaje.

La identidad social es el autoconcepto que se atribuye en un sentido de pertenencia a través de las emociones y valores que se incorporan al mismo sujeto. rrollo de la identidad emergente.

Gur. 2014. Turquía.

Los posibles éxitos y fracasos, el nivel e ingresos, el medio ambiente, la familia, la escuela y los maestros son aspectos que juegan un papel importante en el desarrollo de la identidad profesional. Los estudiantes comienzan a hacer planes para sus profesiones bajo la influencia de héroes o modelos vistos en la familia y/o la televisión.
La identidad se refiere a lo que somos y la forma en la que nos relacionamos con otras personas, formándose con la acumulación de experiencias e interacciones sociales. 


\begin{tabular}{|c|c|c|}
\hline Autor, año, contexto & Principales resultados & Concepto identidad \\
\hline $\begin{array}{l}\text { Kovalcikiene \& } \\
\text { Buksnyte-Marmiene. } \\
\text { 2015. Lituania. }\end{array}$ & $\begin{array}{l}\text { El rol de los estudiantes de doctorado está } \\
\text { más orientado hacia la práctica, siendo } \\
\text { junto con la enseñanza, la experiencia más } \\
\text { significativa para la identidad profesional. }\end{array}$ & $\begin{array}{l}\text { La identidad profesional se compo- } \\
\text { ne de aspectos como expectativas, } \\
\text { interacciones sociales y los roles } \\
\text { que interpretar nuevas tareas y ex- } \\
\text { periencias. }\end{array}$ \\
\hline $\begin{array}{l}\text { Pardal et al. } 2015 . \\
\text { Portugal. }\end{array}$ & $\begin{array}{l}\text { Para la construcción de la identidad docen- } \\
\text { te, las representaciones de los estudiantes } \\
\text { no se pueden separar del contenido y la es- } \\
\text { tructura de su trabajo, resultado del aspec- } \\
\text { to social, de la dinámica de los maestros, la } \\
\text { formación específica y el grupo que ejerce } \\
\text { la misma profesión docente. }\end{array}$ & $\begin{array}{l}\text { La identidad se caracteriza por un } \\
\text { aspecto cultural mediante la atribu- } \\
\text { ción de significados y un enfoque } \\
\text { institucional que da una "identidad } \\
\text { oficial". }\end{array}$ \\
\hline $\begin{array}{l}\text { Zare-ee \& Ghasedi. } \\
\text { 2014. Irán. }\end{array}$ & $\begin{array}{l}\text { La falta de apoyo administrativo además de } \\
\text { la enseñanza de idiomas de manera tradi- } \\
\text { cional, son las principales limitaciones para } \\
\text { el desarrollo de la identidad profesional. Se } \\
\text { necesita cambiar el papel que desempeñan } \\
\text { los profesores que sirven como modelo en } \\
\text { niveles educativos inferiores. }\end{array}$ & $\begin{array}{l}\text { La identidad profesional es un con- } \\
\text { cepto complejo en el que se asocian } \\
\text { aspectos profesionales, sociales y } \\
\text { personales, siendo la definición de } \\
\text { sí mismo y los diferentes roles so- } \\
\text { ciales y familiares lo que predicen el } \\
\text { tipo de desempeño profesional. }\end{array}$ \\
\hline
\end{tabular}

Fuente: elaboración propia.

El desarrollo de la identidad para los estudiantes universitarios constituye la oportunidad de definirse a sí mismos mediante la apropiación de un proyecto que a nivel individual representa la oportunidad de generar un sentido de pertenencia hacía la propia institución que habrá de concederle una identidad, sin embargo, esto no sería posible si no hablamos del proceso colectivo que implica la configuración identitaria, dado que el contexto juega un papel fundamental en la consolidación de la misma, ya que es en el colectivo donde los estudiantes pueden representarse en relación a los otros, a través de las experiencias vividas dentro del aula, en los roles que cada actor social tiene dentro del contexto educativo y en donde los compañeros favorecen las experiencias de aprendizaje, de tal manera que el docente comienza a ser visto como un modelo en el imaginario colectivo, por esta razón, los actores implicados durante el proceso de formación representan roles determinados que habrán de significar las experiencias hacia una de las principales inquietudes de los estudiantes en la etapa de formación, el responder ¿quién soy?.

En todo caso, podemos decir que la etapa de formación representa un periodo fundamental para el desarrollo de la identidad, de tal manera que, la universidad y los sujetos que interaccionan con el estudiante le influyen, determinando las actividades que desempeñará, por ello, se señala que al integrarse socialmente a un grupo se admiten formas de comportamientos, compartiendo significados, creencias y expectativas que el mismo grupo posee, adquiriendo experiencias profesionales y personales, por lo que se determina el rol profesional y se acerca a una consolidación de la identidad profesional (Martín, Conde \& Mayor, 2014).

Con relación a la metodología planteada en los estudios revisados y la tendencia orientada hacia la elección de enfoques de tipo cualitativo, resulta interesante tal elección puesto que los resultados obtenidos en dichos estudios coinciden en señalar a la identidad como un proceso continuo que se muestra heterogénea ya que, para la conformación de la identidad profesional, entran en juego diferentes aspectos como la historia de vida, vista en las experiencias previas y el autoconcepto que va formando el individuo a través de imaginarios y representaciones sociales creando expectativas que habrán de contrastar con los primeros contactos en el campo laboral (Balduzzi y Egle, 2010; Bedacarratx, 2012; Sayago, Chacón \& Rojas, 2008; Gur, 2014; Kim et al., 2013 y Timostsuk \& Ugaste, 2010). 
Además, las relaciones que se forjan dentro y fuera del contexto universitario y la interacción social en las mismas, destacan la influencia del papel que desempeñan tanto los docentes como los compañeros de escuela en el desarrollo de la identidad profesional a través del aprendizaje cooperativo y la influencia de la propia facultad (Limberg et al., 2013; Sugimura \& Shimizu, 2011).

Con relación a lo anterior, cabría señalar que, aspectos como la disciplina, la actividad profesional y el proyecto personal, favorecen la identidad institucional, la cual se construye a partir de las identificaciones con diferentes modelos cuya influencia depende de un momento histórico determinado dentro del contexto donde se desenvuelva el sujeto, obedeciendo a razones ideológicas; así, la apropiación de los atributos de la profesión y de los conocimientos se logra desde el aspecto personal que permite la diferenciación y la autenticidad, siendo entonces el proceso de formación determinante para la construcción de la identidad (Zanatta, Yurén \& Faz, 2010).

Por otro lado, en la Tabla 3, se presentan los estudios realizados en docentes dentro del contexto universitario, presentando en ella los objetivos y la metodología empleada por los autores citados para comprender el proceso de construcción de la identidad profesional.

\section{Tabla 3}

Investigaciones de la identidad profesional en docentes: objetivos y metodología.

\begin{tabular}{|c|c|c|}
\hline $\begin{array}{l}\text { Autor, año, } \\
\text { contexto }\end{array}$ & Objetivos & $\begin{array}{l}\text { Tipo de estudio/Herramientas para } \\
\text { recolección de datos }\end{array}$ \\
\hline $\begin{array}{l}\text { Monereo \& } \\
\text { Domínguez. } 2014 . \\
\text { España. }\end{array}$ & $\begin{array}{l}\text { Identificar los componentes de la identidad do- } \\
\text { cente en relación a su representación sobre su rol } \\
\text { y sobre sus sentimientos respecto a su experiencia. }\end{array}$ & $\begin{array}{l}\text { Estudio cualitativo. Descriptivo-inter- } \\
\text { pretativo/ Entrevista en profundidad. }\end{array}$ \\
\hline $\begin{array}{l}\text { Veiravé, Ojeda, } \\
\text { Nuñez \& Delgado. } \\
\text { 2006. Argentina. }\end{array}$ & $\begin{array}{l}\text { Comprender como elaboran y construyen su tra- } \\
\text { bajo como profesores y como han adquirido su } \\
\text { condición de profesor: identidad profesional. }\end{array}$ & $\begin{array}{l}\text { Estudio cualitativo con historias de } \\
\text { vida/ Entrevistas en profundidad. Bio- } \\
\text { grafías profesionales }\end{array}$ \\
\hline Basei. 2012. Brasil. & $\begin{array}{l}\text { Comprender la construcción de la identidad del } \\
\text { profesor de educación superior durante su carrera } \\
\text { y el desarrollo profesional. }\end{array}$ & $\begin{array}{l}\text { Estudio cualitativo de corte etnográ- } \\
\text { fico/ Entrevista semiestructurada, } \\
\text { observación participante y análisis de } \\
\text { documentos. }\end{array}$ \\
\hline $\begin{array}{l}\text { Contreras, } \\
\text { Monereo \& Badia. } \\
\text { 2010. España. }\end{array}$ & $\begin{array}{l}\text { "Analizar los componentes emergentes en la iden- } \\
\text { tidad docente de los profesores universitarios que } \\
\text { realizan clases en la formación inicial docente, así } \\
\text { como los incidentes críticos que han enfrentado } \\
\text { en su experiencia como formadores en pregrado". }\end{array}$ & $\begin{array}{l}\text { Estudio cualitativo. Descriptivo-in- } \\
\text { terpretativo/ Entrevista semiestructu- } \\
\text { rada, Análisis con programa Atlas. Ti } \\
\text { (Muhr, s.f.). }\end{array}$ \\
\hline $\begin{array}{l}\text { Smit, Fritz \& } \\
\text { Mabalane. } 2010 \text {. } \\
\text { Sudáfrica. }\end{array}$ & $\begin{array}{l}\text { Analizar como los maestros forjan la identidad en } \\
\text { su ambiente de trabajo. }\end{array}$ & $\begin{array}{l}\text { Estudio de caso etnográfico con ele- } \\
\text { mentos de la narrativa/ Diarios de } \\
\text { campo, observación, entrevista narra- } \\
\text { tiva, conversación informal, revisión } \\
\text { de la misión y visión y revistas de las } \\
\text { escuelas }\end{array}$ \\
\hline $\begin{array}{l}\text { Skelton. } 2012 . \\
\text { Inglaterra. }\end{array}$ & $\begin{array}{l}\text { Explorar las experiencias y perspectivas del desa- } \\
\text { rrollo de la identidad en la escuela superior en una } \\
\text { universidad de Inglaterra. }\end{array}$ & $\begin{array}{l}\text { Investigación cualitativa con enfoque } \\
\text { critico interpretativo/Entrevista se- } \\
\text { miestructurada. }\end{array}$ \\
\hline $\begin{array}{l}\text { Martín, Conde } \\
\text { \& Mayor. } 2014 . \\
\text { España. }\end{array}$ & $\begin{array}{l}\text { Detectar que aspectos marcan la construcción } \\
\text { de la identidad profesional del profesorado novel } \\
\text { participante del Instituto de Ciencias de la Educa- } \\
\text { ción de la Universidad de Sevilla. }\end{array}$ & $\begin{array}{l}\text { Informes de los ciclos de supervisión } \\
\text { o ciclos de mejora, los cuales incluyen } \\
\text { observación/grabación, revisión y aná- } \\
\text { lisis. }\end{array}$ \\
\hline $\begin{array}{l}\text { Yesilbursa. } 2012 . \\
\text { Turquía. }\end{array}$ & $\begin{array}{l}\text { Examinar como los profesores de inglés de la Uni- } \\
\text { versidad Turca perciben su identidad del rol pro- } \\
\text { fesional por medio de la metáfora. }\end{array}$ & $\begin{array}{l}\text { Enfoque cualitativo/ Entrevista se- } \\
\text { miestructurada para recolección de } \\
\text { datos demográficos y uso de metáfora } \\
\text { con pregunta específica. }\end{array}$ \\
\hline
\end{tabular}

Fuente: elaboración propia. 
Teniendo como principal actor social la figura del docente para los estudios presentados, podemos inferir el papel fundamental que representa el desarrollo de su trabajo para la configuración identitaria, ya que es a través, principalmente de la percepción de su rol profesional; que se va consolidando la representación que de sí mismo tiene y de la carrera que ha elegido desempeñar, a través de las experiencias y las perspectivas que el trabajo continuo y las expectativas que su misma profesión le generan. De este modo, se vuelve a destacar la importancia de la escuela como un espacio favorecedor hacía el desarrollo de la identidad profesional.

De igual modo, en los estudios realizados con docentes hacia el reconocimiento de la identidad profesional, los estudios cualitativos a través de sus enfoques descriptivo e interpretativo constituyen las opciones más utilizadas para este tipo de investigaciones, destacando nuevamente el uso de entrevistas, aunque es importante mencionar, que con estudios realizados con docentes se ofrecen alternativas más orientadas hacia la construcción de biografías o el uso de la historia de vida a través de la narrativa como opciones metodológicas, lo cual es importante ya que es mediante la capacidad discursiva de los actores involucrados que se puede comprender desde los aspectos individuales como colectivos.

En la intimidad de un relato se le da al sujeto la oportunidad de ir reflexionando acerca de sí mismo, de lo que ha construido y de lo que se ha acercado al poder definir quién es, un relato permite además ir comprendiendo la propia historia a medida que se va evocando, de esta manera, uno o varios relatos favorecen la interpretación del fenómeno que se está investigando y si esto no termina por ayudar a valorar un fenómeno podemos recurrir al encuentro individuo-individuo, en donde, a través de la entrevista $u$ otra herramienta similar, se permite consolidar no solo la visión del propio sujeto que es quien narra, sino del entrevistador que favorece esa narrativa y que a su vez respalda esa interpretación de la realidad de la persona, de esta manera se van construyendo las historias de vida, ayudando a comprender el fenómeno de la identidad profesional.

Para complementar, enseguida se presentan los principales resultados obtenidos en los estudios efectuados en docentes y las características en torno al concepto de identidad que manejan los autores en la Tabla 4. 
Tabla 4

Investigaciones de la identidad profesional en docentes: resultados y concepto de identidad.

\begin{tabular}{ll}
\hline \multicolumn{1}{c}{$\begin{array}{c}\text { Autor, año, } \\
\text { contexto }\end{array}$} & \multicolumn{1}{c}{ Principales resultados } \\
\hline $\begin{array}{l}\text { Monereo \& } \\
\text { Domínguez. } \\
\text { 2014. España. }\end{array}$ & $\begin{array}{l}\text { El rol profesional representa un estado de satis- } \\
\text { facción en donde el individuo avanza hacía la } \\
\text { realización personal, fundamentalmente, en un } \\
\text { proceso de desarrollo profesional que se presen- } \\
\text { ta de forma continua a partir de la elección de } \\
\text { su carrera. }\end{array}$ \\
\hline $\begin{array}{l}\text { Veiravé et al. } \\
\text { 2006. Argentina. }\end{array}$ & $\begin{array}{l}\text { La identidad se construye a partir de la interac- } \\
\text { cióntre diferentes elementos que van desde } \\
\text { los aspectos personales como ideas, creencias y } \\
\text { valores, hasta las experiencias vividas en el des- } \\
\text { empeño de su profesión. }\end{array}$
\end{tabular}

Basei. 2012. La constitución de la identidad se ve influenciaBrasil. da por aspectos como la elección de la carrera, la historia de vida y la formación inicial como el espacio que presenta las primeras bases para la construcción de identidad en los docentes.

\section{Concepto identidad}

La identidad profesional se define por las representaciones que tienen los docentes de sus funciones, sus estrategias de enseñanza y evaluación y sus sentimientos en relación a su práctica profesional.

La identidad profesional se integra a partir de referentes sociales en un espacio de tiempo determinado, donde se hacen presentes las ideologías que se van generando con relación a la profesión a través de las experiencias personales.

La identidad no es algo fijo ni inmutable, se compone de las experiencias vividas, de los sentimientos y necesidades del sujeto y de las relaciones que establece con el entorno configurando una definición de su rol profesional.

Contreras et al. Los componentes en la identidad docente in2010. España. cluyen el enseñar y aprender en la universidad, las metodologías y estrategias en las clases, los sentimientos asociados a la docencia y la formación permanente del profesorado universitario. Aunque los docentes en este estudio se definen a sí mismos como sujetos que se han construido en solitario siguiendo una formación informal.

Smit et al. 2010. La formación de la identidad profesional del doSudáfrica. cente tiene lugar en el espacio educativo, siendo facilitada por las actividades en donde hay interacción y las relaciones que se establecen dentro de este espacio.

Skelton. 2012. Surgieron tres identidades docentes en esta inInglaterra. vestigación: "especialistas de enseñanza", "profesionales mezclados" y "los investigadores que enseñan"; lo que sugiere diferentes trayectorias para el desarrollo de la enseñanza.

Martín et al. Existen cuatro dimensiones (emocional, social, 2014. España. didáctico-pedagógica e institucional-administrativa) en las que se sustenta la construcción de la identidad profesional docente, siendo la dimensión emocional transversal a todas las demás; siendo además, el contexto, un elemento sustancial en el proceso de consolidación de la identidad profesional.

Yesilbursa. 2012. El uso de la metáfora permite obtener inforTurquía. mación relevante acerca de cómo los docentes se ven a sí mismos como profesionales, aunque en general los docentes no reflexionan respecto a su identidad del rol profesional por lo que el uso de múltiples metáforas podría describirla totalmente.
La identidad es definida partir de una serie de interrogantes que evalúan el ciones y creencias en relación al actuar profesional orientando así, la conducta hacía lo que se tiene que hacer, de tal manera que se pueda puntualizar el rol profesional, la percepción del aprendizaje adquirido y los sentimientos asociados a su profesión.

La identidad surge de las experiencias de vida y de la interacción entre el contexto laboral y los significados, valores y discursos que se comparten en este contexto.

La identidad se constituye por la interacción compleja entre las micro, meso y macro influencias, siendo mediada por los factores internos del sujeto.

La identidad es un proceso continuo que se define a partir de los rasgos de personalidad, las motivaciones y las actitudes del sujeto, a partir de la interacción dada con un grupo profesional en un contexto determinado.

La identidad del rol profesional esta visto como un proceso dinámico que está en contradicción y conflicto, definido por los diferentes roles que adoptan los profesores a través de las interacciones con estudiantes y colegas. ¿Quién soy? A partir de las significa-

Fuente: elaboración propia. 
La identidad profesional para los docentes, implica un proceso continuo en donde intervienen factores internos como los rasgos de personalidad, así como, los sentimientos y valores en relación a su profesión, de esta manera, la historia de vida resulta crucial en el entendimiento de la representación social que su profesión le confiere, recuperando las experiencias de vida dentro y fuera del espacio educativo; nuevamente observamos en el contexto, una esfera en donde el rol profesional viene a consolidarse principalmente a partir de dos aspectos: la elección profesional que el docente realiza en su formación inicial y las interacciones sociales que representan la oportunidad de significarse en relación a los y por los otros, a fin de que el docente pueda simbolizar los significados en relación a su trabajo para entender el quién es a partir de lo qué tiene que hacer y porqué.

De lo anteriormente expuesto, se desprende que en la misma forma en la que predomina el enfoque cualitativo como método de estudio en estudiantes universitarios, se acentúa dicha metodología en estudios realizados con docentes, siendo todos ellos de carácter cualitativo y la entrevista semiestructurada la herramienta de recolección de datos más utilizada; a lo anterior se añade el análisis de documentos y la observación videograbada como alternativas complementarias para el cumplimiento de los objetivos planteados en dichos estudios.

Además se destaca la historia de vida y el proceso de formación continua como factores clave para la comprensión de la construcción de la identidad, es por ello que, dependiendo del lugar que ocupe el sujeto, la función que cumpla, sus tareas y las actividades que le sean asignadas y autoasignadas durante el proceso de formación, es como irá desarrollando su identidad profesional (Basei, 2012; Monereo \& Domínguez, 2014; Veiravé et al., 2006), igualmente, el rol profesional atañe a un sentido de auto percepción que tienen los docentes vistos como modelos dentro del entorno educativo lo que implica que "son un reflejo de lo que quieren que sus alumnos piensen, hagan y sean" (Contreras et al., 2010).
Lo anterior admite la necesidad de reforzar la eficiencia del proceso enseñanza-aprendizaje con la convicción de que no se trata solo de formar buenos profesionales, sino de entender que en el desarrollo de la identidad, la labor docente representa la capacidad de generar una serie de transformaciones que van más allá de la propia profesión, ampliando el reconocimiento social de la misma y recuperando el sentido emocional que representa una elección vocacional que surge de los valores y creencias forjados desde la infancia y que continúa a través de su trayectoria personal (Contreras et al., 2010; Gur, 2014; Skelton, 2012; Smit et al., 2010).

Del análisis precedente, de las definiciones referidas por los autores en torno al concepto de identidad, se resalta la necesidad de significar dicho proceso, dado que en su mayoría no se encuentra una definición explicita que permita representar la importancia del estudio en torno a la identidad profesional, por lo que se propone la siguiente definición:

La identidad es una construcción social que le permite al sujeto definirse a sí mismo mediante un proceso de diferenciación $y$ apropiación de formas socialmente reconocidas, producto de la interacción social dada entre el individuo y los otros; lo que facilita la representación de su rol profesional en un grupo de pertenencia dentro un marco de realidad determinada.

El responder a nuestra identidad en el sentido de quiénes somos, implica una definición de lo que no somos, lo que nos incluye y al mismo tiempo nos excluye de los demás, así, el sujeto al adoptar y recrear imaginarios y simbólicos respecto a lo que es, lo que hace y lo que cree, da un sentido a sí mismo que le permite acercarse al reconocimiento social que una profesión le confiere.

La construcción de la identidad profesional dentro del contexto universitario se constituye a partir de los referentes mostrados en el siguiente esquema. 


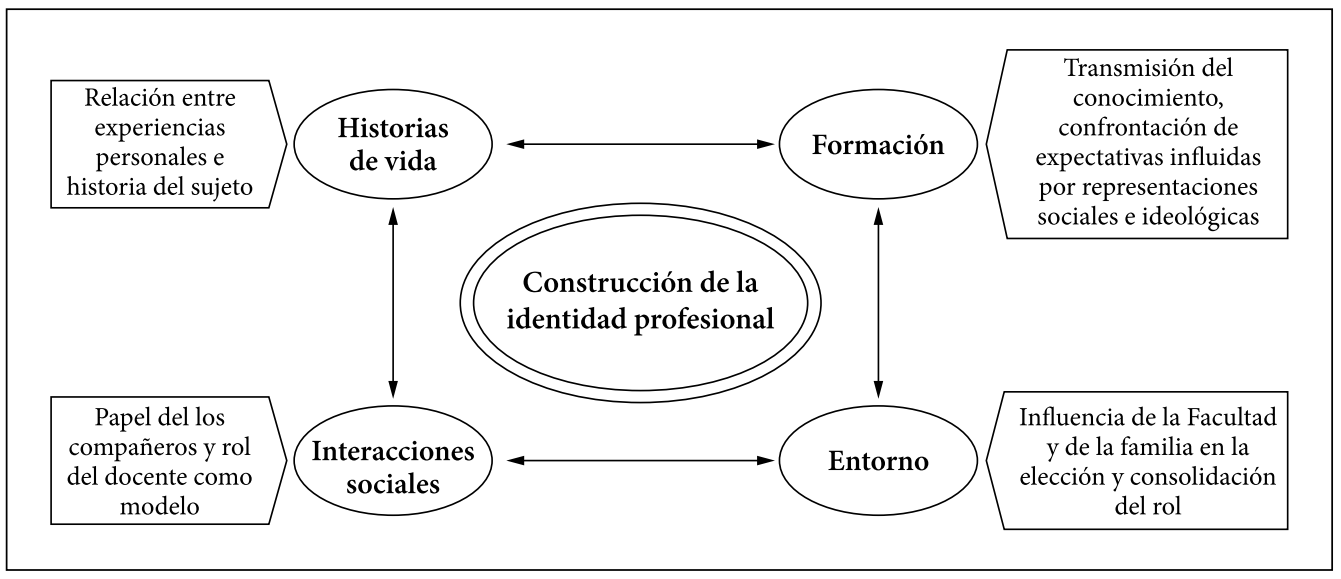

Figura 1. Elementos principales para la construcción de la identidad profesional.

Fuente: elaboración propia

La construcción de la identidad profesional se va desarrollando a partir de la interrelación de los elementos anteriormente mostrados (Figura 1), a través de la historia de vida del sujeto, se van recuperando las experiencias personales y las interacciones sociales que va consolidando en los diferentes espacios donde se ubica, ya sea en el entorno institucional o en el familiar, interacciones que hablan de la forma de relacionarse con el otro a partir del sí mismo, de esta manera, si bien es cierto el compañero se vuelve un acompañante en el proceso de configuración identitaria, es el docente en el que recae el papel principal al ser la representación natural del rol profesional, por ello el desarrollo de la identidad profesional se ve favorecido.

Como entorno educativo y en búsqueda de la consolidación del rol profesional, esto no sería posible si no atendemos a la etapa de formación como un referente en donde se va logrando una representación del sí mismo al identificarse con personas, valores y comportamientos precisamente a través de la representación de la propia profesión integrando factores personales y sociales que se perciben como propios y que dan estabilidad y continuidad a la vida profesional (Monereo \& Domínguez, 2014); es en esta etapa donde precisamente las expectativas hacen eco al desarrollarse en un escenario instaurado por el propio contexto educativo, dándose una confrontación con lo que se espera y lo que en realidad es.

\section{CONCLUSIONES}

En atención a la revisión expuesta y en referencia a las vertientes perseguidas en este trabajo, la relación entre los objetivos de los diferentes estudios y las metodologías utilizadas, indican que la metodología cualitativa constituye un enfoque propicio para responder a necesidades que buscan comprender el proceso de construcción de la identidad profesional, dado que se centra en la comprensión de la subjetividad y significados que los individuos elaboran, considerando que la realidad es construida por las personas involucradas en la situación que se estudia, pudiendo tener puntos de coincidencia, de esta manera, el enfoque cualitativo permite analizar experiencias textuales de los protagonistas de un fenómeno y comprender lo que hacen, piensan y dicen mediante la observación realizada en su contexto (Bedacarratx, 2012).

Es por ello que la consolidación de la identidad se logra con el descubrimiento que el profesional realiza de sus ideas básicas y creencias acerca de sí mismo, la situación con los demás profesionales, las concepciones y autopercepciones en torno a la tarea que realiza o a su rol profesional (Hirsch, 2013), retomando la interacción con otros actores y contextos, otorgándole un sentido más amplio y un significado más profundo; a través de una reconstrucción de la historia de vida, la identificación y diferenciación con otras disciplinas y la consoli- 
dación o crisis de identidad, dándose dicho proceso de construcción a partir de la delimitación del objeto de estudio y de la diferenciación con otras disciplinas (Zanatta, Yurén \& Faz, 2010); por lo cual el sujeto no terminaría de definirse a sí mismo sin una clara representación de lo que su profesión le concede y de lo que la vuelve diferente a las demás.

Debe señalarse que el proceso de construcción de una identidad profesional comienza a partir de la infancia, en donde como estudiantes de educación básica, los sujetos van formando un sistema de valores, creencias y elecciones que correlacionan con su entorno, se espera entonces que durante la formación universitaria los estudiantes desarrollen una identidad propia a partir de la construcción del yo ideal (Basei, 2012) y que los docentes maduren sus identidades profesionales; de esta manera, los sujetos serán capaces de consolidar tal sistema en la medida en la que se fortalezcan sus vínculos personales, teniendo en el lenguaje la herramienta ideal a través de la cual el ser humano aumenta la capacidad de relacionarse con los otros, al mismo tiempo que le permite reconocerse a sí mismo, construyendo así la propia identidad al comprender quién es y lo más importante quién NO es.

El responder a esta pregunta, ¿Quién soy?, es lo que permite comprender el proceso de construcción de la identidad como algo permanente, que se va deconstruyendo y reconstruyendo a medida que el ser humano va generando más relaciones, cuando su interacción con los otros le permite generar nuevas historias, de esta manera entendemos que la persona no es la misma, aunque hiciera las mismas cosas, con las mismas personas, siempre será una historia diferente, de aquí la dificultad de entender y definir la identidad de una persona; si bien es cierto es improbable que una definición de la identidad pueda ser inmutable, la realidad nos indica que a través de la interpretación de la propia vida podemos acercarnos a reconocer quiénes somos y cuál es el sentido de nuestro quehacer profesional, siendo esta la gran aportación del enfoque cualitativo que nos permite reconstruir el significado que la etapa de formación profesional tiene para la configuración identitaria.

\section{REFERENCIAS BIBLIOGRÁFICAS}

Balduzzi, M. \& Egle, R. (2010). Representaciones sociales e ideología en la construcción de la identidad profesional de estudiantes universitarios avanzados. Revista Intercontinental de Psicología y Educación, 12(2), 65-83. Disponible en: https://www. redalyc.org/html/802/80218376004/

Barrenetxea, M., Cardona, A., Barandiaran, M., Mijangos, J. \& Olaskoaga, J. (2013). El desarrollo de la identidad profesional en la universidad: una propuesta desde la docencia en Gestión de Negocios. Revista de Docencia Universitaria, 11(2), 413-441. Disponible en: https://dialnet.unirioja.es/ descarga/articulo/4414695.pdf

Basei, A. (2012). O desenvolvimento professionale a construcão da identidade do proesor de educação física do ensino superior. Revista Mackenzie de Educação Física e Esporte, 11(1), 44-60. Disponible en: http:// editorarevistas.mackenzie.br/index.php/ remef/article/view/2603

Bedacarratx, V. (2012). Futuros maestros y construcción de una identidad profesional: una mirada psicosocial a los procesos que se ponen en juego en los trayectos de formación en la práctica. Revista Electrónica de Investigación Educativa, 14(2), 133149. Disponible en: https://www.redalyc. org/pdf/155/15525013010.pdf

Contreras, C., Monereo, C. \& Badia, A. (2010). Explorando en la identidad: ¿Cómo enfrentan los docentes universitarios los incidentes críticos que ocurren en las aulas de formación de futuros profesores? Estudios Pedagógicos, XXXVI(2), 63-81. Disponible en: https://scielo.conicyt.cl/scielo.php?script=sci arttext\&pid $=$ S0718-07052010000200004

Gur, T. (2014). A discourse analysis: Professional identity development of language teacher candidates. Educational Research and Reviews, 9(15), 510-515. Disponible en: http://www.academicjournals.org/app/ webroot/article/article1405589329 Gur. pdf 
Hirsch, A. (2013). Elementos teóricos y empíricos acerca de la identidad profesional en el ámbito universitario. Perfiles Educativos, 35(140), 63-82.

Kim, C., Copeland, H., Hopson, R., Simmons, T. \& Leibowitz, M. (2013). The role of professional identity in graduate school success for under-represented minority students. Biochemistry and Molecular Biology Education, 41(2), 70-75. Disponible en: https://iubmb.onlinelibrary.wiley. com/doi/pdf/10.1002/bmb.20673

Kovalcikiene, K. \& Buksnyte-Marmiene, L. (2015). Towards an understanding of doctoral students' professional identity complexity. Procedia-Social and Behavioral Sciences, 191, 2693-2698. Disponible en: https://www.sciencedirect.com/science/article/pii/S1877042815028633

Limberg, D., Bell, H., Super, J., Jacobson, L., Fox, J., DePue, M., ... Lambie, G. (2013). Professional identity development of counselor education doctoral students: a qualitative investigation. The Professional Counselor, 3(1), 40-53. Disponible en: https://eric.ed.gov/?id=EJ1063181

Martín, A., Conde, J. \& Mayor, C. (2014). La identidad profesional docente del profesorado novel universitario. REDU. Revista de docencia universitaria, 12(4), 141-160. Disponible en: https://polipapers.upv.es/ index.php/REDU/article/view/5618

Monereo, C. \& Domínguez, C. (2014). La identidad docente de los profesores universitarios competentes. Educación XX1, 17(2), 83-104. Disponible en: http:// repositorio.minedu.gob.pe/bitstream/ handle/123456789/3074/La\%20identidad\%20docente\%20de\%20los\%20profesores\%20universitarios\%20competentes. pdf? sequence $=1$ \&isAllowed $=y$

Navarrete, Z. (2008). Construcción de una identidad profesional. Revista Mexicana de Investigación Educativa, 13(36), 143-171. Disponible en: https://www.researchgate. net/publication/28208951 Construccion de una identidad profesional Los pedagogos de la Universidad $\mathrm{Na}$ cional Autonoma de Mexico y de la Universidad Veracruzana
Pardal, L., Neto-Mendes, A., Martins, A. \& Goncalves, M. (2015). Identities and structure of teacher's work: a representation. Procedia-Social and Behavioral Sciences, 185, 165-171. Disponible en: https://www.researchgate.net/publication/277948074 Identities and Structure of Teacher's Work A Representation

Sayago, Z., Chacón, M. \& Rojas, M. (2008). Construcción de la identidad profesional docente en estudiantes universitarios. $E D U$ CERE-Investigación arbitrada, 42(12), 551-561. Disponible en: https://www.redalyc.org/html/356/35614569016/

Skelton, A. (2012). Teacher identities in a research-led institution: in the ascendancy or on the retreat? British Educational Research Journal, 38(1), 23-39. Disponible en: https://www.tandfonline.com/doi/ab s/10.1080/01411926.2010.523454

Smit, B., Fritz, E. \& Mabalane, V. (2010). A conversation of teachers: in search of professional identity. The Australian Educational Researcher, 37(2), 93-106. Disponible en: https://link.springer.com/ article/10.1007/BF03216924

Sugimura, K. \& Shimizu, N. (2011). Identity development in the learning sphere among Japanese first-year university students. Child Youth Care Forum, 40, 25-41. Disponible en: https://www.researchgate. net/publication/225578609 Identity Development in the Learning Sphere Among Japanese First-Year University Students

Timostsuk, I. \& Ugaste, A. (2010). Student teachers' professional identity. Teaching and Teacher Education, 26, 1563-1570. Disponible en: https://www.sciencedirect.com/ journal/teaching-and-teacher-education/ vol/26/issue $/ 8$

Veiravé, D., Ojeda, M., Nuñez, C. \& Delgado, P. (2006). La construcción de la identidad de los profesores de enseñanza media. Biografías de profesores. Revista Iberoamericana de Educación, 40(3), 1-11. Disponible en: https://rieoei.org/RIE/article/view/2512 
González P., Marín, R. \& Soto, MA. (2019). La identidad profesional en estudiantes y docentes desde el contexto universitario: una revisión. Revista Ciencias de la Actividad Física UCM, $\mathrm{N}^{\circ}$ 20(1), enero-junio, 1-14. DOI: http://doi.org/10.29035/rcaf.20.1.4

Yesilbursa, A. (2012). Using a metaphor to explore the profesional role identities of higher education English language instructors. Procedia-Social and Behavioral Sciences, 46, 468-472. Disponible en: https:// ac.els-cdn.com/S1877042812012724/1s2.0-S1877042812012724-main.pdf? tid=a63690a7-95fe-44d5-8501-7b4243d6 $\underline{181 \mathrm{e} \& \mathrm{acd} n \mathrm{at}=155311290698572 \mathrm{fc} 5 \mathrm{f} 00}$ $\underline{94572 \mathrm{cfd} 6 \mathrm{cc} 0 \mathrm{a} 6882 \mathrm{~d} 2 \mathrm{f} 4}$

Zanatta, E., Yurén, T. \& Faz, J. (2010). Las esferas de la identidad disciplinar, profesional e institucional en la universidad pública mexicana. Argumentos UAM-X, 62, 87104. Disponible en: http://www.scielo. org.mx/scielo.php?script=sci arttext\&pi $\mathrm{d}=$ S0187-57952010000100004

Zare-ee, A. \& Ghasedi, F. (2014). Professional identity construction issues in becoming an english teacher. Procedia-Social and Behavioral Sciences, 98, 1991-1995. Disponible en: https://www.researchgate. net/publication/264973840 Professional Identity Construction Issues in Becoming an English Teacher

Dirección para correspondencia

Paulina Elizabeth González Orozco

Universidad Autónoma de Chihuahua

Maestría (Candidato a grado Doctorado)

México

Chihuahua, Chihuahua, C.P. 31125

No ORCID: 0000-0003-1109-6098

Contacto:

psic.eligonzalez@gmail.com

Recibido: : 06-11-2018

Aceptado: 03-01-2019 